\title{
Ormosil-Coated Conjugated Polymers for the Detection of Explosives in Aqueous Environments
}

Ross N. Gillanders, Iain A. Campbell, James M.E. Glackin, Ifor D.W Samuel, Graham A. Turnbull*

Organic Semiconductor Centre, SUPA, School of Physics \& Astronomy, University of St Andrews, Fife KY16 9SS, Scotland

\begin{abstract}
A fluorescence-based sensor for detecting explosives, based on a conjugated polymer coated with an ormosil layer, has been developed for use in aqueous environments. The conjugated polymer Super Yellow was spin-coated onto glass substrates prior to a further spin-coating of an MTEOS/TFP-TMOS-based ormosil film, giving an inexpensive, solution-based barrier material for ruggedization of the polymer to an aqueous environment. The sensors showed good sensitivity to 2,4-DNT in the aqueous phase at micromolar and millimolar concentrations, and also showed good recovery of fluorescence when the explosive was removed.
\end{abstract}

Keywords: Organic semiconductors, sol-gels, aqueous explosives, optical sensing, super yellow

Email: gat@st-andrews.ac.uk 


\section{Introduction}

Conjugated polymers are highly-emissive materials commonly used as the active layer in devices including organic LEDs (OLEDs), polymer lasers, and optical sensors [1-6]. Due to photooxidation and mechanical degradation of the polymers, OLEDs and lasers normally require encapsulation in epoxy layers to prevent ingress of water and oxygen, and so extend the operational lifetime. For sensor layers, this approach is not suitable since the layer requires direct contact with the analyte. Optical sensing films are often needed to operate in aggressive environments, including, for instance, water flows or humid outdoor environments. However, to deploy these polymer materials, a robust, optically clear, porous layer would be necessary to allow analyte ingress while protecting the polymer layer itself.

Sol-gel materials are well-known matrices in optical sensor fabrication, due to advantages in solution-processing, optical clarity, suitability for aqueous deployment and long-term stability. Organically modifying the sol-gel with a precursor to create an organically-modified silicate (ormosil) can, amongst other benefits, increase the hydrophobicity of the sol-gel [7, 8]. These properties make sol-gels attractive coating layers for the polymers to mitigate potential weaknesses in real-world deployment. By coating the polymer films with an ormosil layer, the film could allow ingress of the explosive molecules while protecting the more sensitive polymer from water.

Conjugated polymers are particularly suited for optical sensing of nitroaromatic molecules for explosives detection [9-14]. When the polymer absorbs light an exciton is formed which emits fluorescence; but electron transfer from the polymer to a sorbed nitroaromatic dissociates the exciton and so can be observed by a decrease in light emission. While these materials show good 
sensitivity to explosives in the vapour phase, practical detection of these compounds in the aqueous phase is more challenging, particularly for the photophysical and mechanical stability of the polymers in such environments. Other methods, such as Surface Plasmon Resonance (SPR) [15], Raman Spectroscopy [16] and electrochemical sensors [17, 18] show good performance in aqueous nitroaromatic detection. However, an easily-processed, optically-clear, and robust protective layer for the conjugated polymers would be of high importance and a promising tool for optical explosives sensing, since the sensor itself can be sensitive, disposable, and reversible. In this paper we demonstrate a hybrid thin-film sensor, made from a conjugated polymer and sol-gel barrier, which can sensitively and reversibly detect the explosive molecule 2,4-DNT in water.

\section{Experimental}

\subsection{Materials}

Triethoxymethylsilane (MTEOS) $\left(\mathrm{CH}_{3} \mathrm{Si}\left(\mathrm{OC}_{2} \mathrm{H}_{5}\right)_{3}\right)$ and Trimethoxy(3,3,3-trifluoropropyl)silane (TFP-TMOS) $\left(\mathrm{C}_{6} \mathrm{H}_{13} \mathrm{~F}_{3} \mathrm{O}_{3} \mathrm{Si}\right)$, 2,4-Dinitrotoluene (2,4-DNT), toluene, ethanol (EtOH) and hydrochloric acid (37\%) $(\mathrm{HCl})$ were purchased from Sigma Aldrich. Poly[\{2,5-di(3,7decyloxy)-1,4- phenylenevinylene-co-\{3-(4'- (3',7"-decyloxy)phenyl)- 1,4-phenylenevinylene $\}$ co-\{3- (3'-(3',7-decyloxy)phenyl)-1,4- phenylenevinylene $\}]$, known as PDY-132 (Super Yellow), was purchased from Merck, and $1 \mathrm{~cm} \mathrm{x} 1 \mathrm{~cm}$ glass cover slips were purchased from Agar Scientific. All chemicals were used as received without further purification.

\subsection{Film fabrication}


Super Yellow films were fabricated by dissolving the polymer in toluene at a concentration of 6.5 $\mathrm{mgml}^{-1}$, prior to spin-coating on a glass cover slip at $2000 \mathrm{rpm}$ for 60 seconds. The ormosil component was made by combining MTEOS, TFP-TMOS, EtOH, water and $\mathrm{HCl}$ in a 1:1:6.25:4:0.007 ratio following the process outlined in [7]; a ratio of 4:1 water:precursor was selected since higher ratios did not appear to allow explosive ingress due to tighter crosslinking producing smaller pores in the ormosil matrix, and lower ratios resulted in lower Photoluminescence Quantum Yield (PLQY) values by an average of 17.9\%. To prepare the ormosil, the silane precursors and EtOH were combined in a vial and set to stir, with subsequent dropwise addition of the $\mathrm{HCl}$. The solution was stirred for one week at ambient temperature prior to spin-coating over the Super Yellow film at $2000 \mathrm{rpm}$ for 60 seconds. The resulting composite was cured on a hotplate at $60^{\circ} \mathrm{C}$ over 24 hours. This temperature was chosen to avoid possible degradation of the PLQY of the Super Yellow film due to chemical oxidation at elevated temperatures, but to still allow sufficient evaporation of the solvent and promote ormosil curing. Lower curing temperatures resulted in films with a lowered PLQY of up to 7\%. Once hotplate curing was complete the composite films were stored at room temperature in the dark in sealed containers until used.

\subsection{Photophysics}

Absorption spectra were recorded on a Varian Cary 300 Bio UV-Vis absorption spectrometer under ambient conditions. Emission spectra were recorded on an Edinburgh Instruments FLS980 Fluorescence spectrometer in steady-state mode with an excitation wavelength of $405 \mathrm{~nm}$ for the uncoated film and $440 \mathrm{~nm}$ for the coated film. PLQY measurements of the ormosil-coated sensors were performed in an integrating sphere [19] using a Hamamatsu Photonics C9920-02 measurement system with an excitation wavelength of $440 \mathrm{~nm}$. A Veeco Dektak 150 surface 
profiler was used to investigate film thicknesses, which were typically $100 \mathrm{~nm}$ for the final layers. The response to nitroaromatics is unaffected by the thickness of the ormosil layer, within the parameter range studied of $100 \mathrm{~nm}-200 \mathrm{~nm}$. Thicker polymer films have been shown [20] to slow the quenching response due to quenching molecules penetrating the polymer matrix at a fixed rate. To measure water contact angle, a goniometer was used with angles estimated by eye using the built-in protractor.

\subsection{Experimental Sensing Procedure}

Fluorescence from the sensor film was measured while immersed in water and various concentrations of 2,4-DNT. The films were excited with light at $440 \mathrm{~nm}$. The spin-coated films were placed in a home-made bracket and inserted into a plastic cuvette to minimise movement during measurement, and to ensure reproducibility of sensor position between measurements. Calibration curves were obtained by taking an emission measurement in deionised water prior to adding solutions of 2,4-DNT varying from $7.2 \mu \mathrm{M}$ to $7.2 \mathrm{~mm}$. To test the recovery and repeatability behaviour of the sensor, measurements were taken as the film was alternately immersed in clean and contaminated water.

To test the response of the films to 2,4-DNT in the vapour phase, the film was placed in a sealed chamber connected to a nitrogen line as described elsewhere [3]. The film was exposed to clean nitrogen prior to introducing 2,4-DNT vapour into the line at a concentration below the saturated vapour pressure of $411 \mathrm{ppb}$ [21], and the emission measured during 100 seconds of exposure. 


\section{Results and Discussion}

The absorbance and emission spectra of an uncoated Super Yellow film, and coated Super Yellow film in air and water are shown in Figure 1. Both spectra show wide bandwidths with the absorbance peak at $405 \mathrm{~nm}$ and the emission peak at $590 \mathrm{~nm}$ for the uncoated Super Yellow films. The ormosil-coated Super Yellow film displays broadened spectra with some spectral shifting, by around $40 \mathrm{~nm}$ to the red in the absorption spectrum, and by $15 \mathrm{~nm}$ to the blue in the emission spectrum. This may be attributable to partial mixing of the polymer and ormosil layers at the interface. The PLQY of an ormosil-coated film was found to be $32 \%$, compared to $40 \%$ for an uncoated film.

Figure 1: Absorbance (green) and emission (navy blue) of uncoated Super Yellow film; absorbance and emission spectra of ormosil-coated Super Yellow (SY) in air (purple and light blue lines respectively), and in water (red and black lines respectively).

Films with mechanically robust properties were obtained by spin-coating and hotplate curing. Figure 2 compares the response to 2,4-DNT, in the vapour phase, of uncoated and ormosil-coated polymer films. Quantitative evaluations for vapour-phase 2,4-DNT detection is outlined in further

detail in reference [3]. The fluorescence is seen to decrease rapidly following the start of the exposure at the same rate for both samples, suggesting that the ormosil layer is not impeding access to the quenching sites. Fluorescence from both films is quenched by approximately $50 \%$ in the 
first 20 seconds of 2,4-DNT exposure, and decrease by $65 \%$ and $63 \%$ over 100 seconds for the uncoated and coated films, respectively.

Figure 2. Comparison in response of uncoated Super Yellow film (black line) and ormosil-coated Super Yellow film (red line) to vapour-phase 2,4-DNT.

Sensing in the aqueous phase is shown in Figure 3a. The concentration of 2,4-DNT was increased from 0 to $7.2 \mathrm{~mm}$ in deionised water in steps of $7.2 \mu \mathrm{M}, 36 \mu \mathrm{M}, 0.36 \mathrm{~mm}, 0.72 \mathrm{mM}, 1.8 \mathrm{mM}$, and 3.6 mM. A decrease in luminescence was seen in proportion with the increasing concentration, and the response can be fitted with a triple exponential decay curve. A low level of residual emission can be observed at the upper concentration of $7.2 \mathrm{~mm}$, suggesting some quenching sites are inaccessible to nitroaromatics. The sensitivity of the film is shown as a Stern Volmer plot in Figure $3 b$ with a ratio $I_{0} / \mathrm{I}$ of 15.5 at $7.2 \mathrm{mM}$. The slope provides information on the quenching constant Ksv via Equation (1):

$$
\frac{I_{0}}{I}=1+\tau_{0} k_{q}[Q]=1+K_{S V}[Q]
$$

where $I_{0}$ is the unquenched emission intensity, I the emission in the presence of quencher [Q], $\tau_{0}$ the fluorescent lifetime in the absence of quencher, and $\mathrm{k}_{\mathrm{q}}$ the bimolecular rate constant.

The Stern-Volmer plot is fairly linear, consistent with the high accessibility of the quenching sites by the nitroaromatic molecules. The quenching constant $\mathrm{K}_{\mathrm{SV}}$ has the value $2000 \mathrm{M}^{-1}$, while the Limit of Detection (LoD) is $8.17 \mu \mathrm{M} / 1.49 \mathrm{ppm}$. This compares well with similar 2,4-DNT sensors in aqueous media [22, 23] and in acetone [24], and is below the Threshold Limit Value (TLV) of 2,4-DNT in water at $8.2 \mu \mathrm{M}[25]$. 
Figure 3. (a) Response of the luminescence from an ormosil-coated Super Yellow film to 0-7.2 mM 2,4-DNT in water; (b) Stern-Volmer plot of the corresponding data; (c) Modified SternVolmer plot of data.

A modified Stern Volmer plot can give a useful graphical representation of the accessible and inaccessible fractions of the quenching sites [26], which is of particular interest in sol-gel based sensing due to the nature of the porous matrix. Briefly, if two quenching populations exist in a sensing film giving two emission intensities, $I_{a}$ (accessible) and $I_{b}$ (inaccessible), then the total observed emission in the absence of quencher is given by Equation (2):

$$
I_{0}=I_{0 a}+I_{0 b}
$$

Since $\mathrm{I}_{\mathrm{b}}$ is not quenched, in the presence of a quencher Equation 1 then becomes Equation (3):

$$
I=\frac{I_{0 a}}{1+K_{a}[Q]}+I_{0 b}
$$

which leads via subtraction and inversion to equation (4):

$$
\frac{I_{0}}{\Delta I}=\frac{1}{f_{a} K_{a}[Q]}+\frac{1}{f_{a}} .
$$

In this way the intercept represents the remaining emission in the presence of a theoretical infinite amount of quencher, therefore $\mathrm{f}_{\mathrm{a}}^{-1}$ provides the fraction of inaccessible sites. Applying this to the 
data from Figure 3a yields a plot shown in Figure 3c with an intercept of 1.06 and a slope of 1.15 $\mathrm{x} 10^{-3}$, corresponding to a fractional accessibility of $94.5 \%$ and $\mathrm{K}_{\mathrm{a}}=780$.

Figure 4. Recovery profile of the ormosil-SY film alternating between $0 \mathrm{~mm}$ and $7.2 \mathrm{~mm}$ 2,4-DNT in deionised water.

Recovery of the sensor film is an important parameter, particularly in cases where the sensor may be left in-situ over a period of time for ongoing monitoring. Figure 4 shows the real-time response of the film with a stepwise change in concentration between 0 and $7.2 \mathrm{mM}$. A recovery to $60 \%$ of the initial light intensity is seen after the first cycle. This is different from Super Yellow sensors operating in the vapour phase which shows no recovery of the fluorescence without application of heat to the film. The recovery observed in water shows that molecules in the interface layer are less strongly bound to the film, possibly due to the water rinsing the explosive molecules from the ormosil pores. Good recovery is seen over the five cycles and compares well with similar results in the literature $[22,27]$. However, we note that in the work by Sun et al [22] the film is removed from the solution and dried in nitrogen prior to reuse, whereas the sensor presented in this work remains in-situ over the full cycle. Similarly, in the work by Beyazkilic et al. [27], the films are dried at room temperature under ambient conditions prior to reuse. The measured contact angle of $90^{\circ}$ suggests that some water is able to penetrate the pores since this value is not sufficiently hydrophobic to completely disallow water ingress, thus allowing some "rinsing" of the pores to remove the 2,4-DNT molecules. This is supported by the fast initial quenching at lower concentrations to around $60 \%$ of $\mathrm{I}_{0}$, shown in Figure $4 \mathrm{a}$, which suggests some 2,4-DNT molecules 
are penetrating deep enough into the ormosil matrix to resist any subsequent rinsing, with the remainder of quenching at higher 2,4-DNT concentrations happening at or near the surface where the molecules can be fully reversibly removed. The cycling between 0 and $7.2 \mathrm{~mm}$ 2,4-DNT shown in Figure 5 shows broad agreement with this, with the intensity cycling between $20 \%$ at $0 \mathrm{~mm} 2,4$ DNT and $60 \%$ at $7.2 \mathrm{~mm} 2,4-\mathrm{DNT}$.

\section{Conclusions}

An optical sensor for the detection of explosive molecules in aqueous solution, based on an ormosil-coated conjugated polymer, has been demonstrated. The sensing film was fabricated by spin-coating a polymer layer, prior to spin-coating with an ormosil layer, and curing the resulting film. The films showed spectral broadening with peak shifts compared to uncoated Super Yellow films indicating some intermixing of the layers. A comparison was made in the vapour phase between coated and uncoated polymers, and the ormosil layers were shown to not impede the ingress of explosive vapours to the fluorescent layer. Sensors were then tested in the aqueous phase between 0 - $7.2 \mathrm{~mm}$ 2,4-DNT in water, showing good recovery between films. The films were shown to be reversible in the aqueous phase, with a modified Stern-Volmer plot estimating around 95\% accessibility of the quenching sites in the polymer layer by the explosive. These results indicate that coating a conjugated polymer in an ormosil layer results in a robust, porous barrier allowing analyte ingress to the sensing layer while providing mechanical protection of the polymer in an aqueous environment. The reversibility and stability provided by the ormosil layers indicate that this could allow long-term deployment applications in aqueous detection of explosives, for 
instance in ports, outflow pipes of suspected drug factories, or in water-logged areas with legacy UXO presence.

\section{Supporting Information}

Data supporting this research can be found at [DOI].

\section{Acknowledgements}

This project has received funding from the European Union's Seventh Framework Programme for research, technological development and demonstration under agreement no 284747 and the EPSRC under EP/K503940/1. IDWS acknowledges a Royal Society Wolfson Research Merit Award.

\section{References}

[1] S.W. Thomas, III, G.D. Joly, T.M. Swager, Chemical sensors based on amplifying fluorescent conjugated polymers, Chemical Reviews 107(4) (2007) 1339-1386.

[2] A. Narayanan, O.P. Varnavski, T.M. Swager, T. Goodson, III, Multiphoton fluorescence quenching of conjugated polymers for TNT detection, Journal of Physical Chemistry C 112(4) (2008) 881-884.

[3] R.N. Gillanders, Samuel, I.D.W., Turnbull, G.A., A Low-Cost, Portable Optical Explosive-vapour Sensor, Sensors and Actuators B-Chemical 245 (2017) 334-340.

[4] M.A. Ali, S.S.Y. Chen, H. Cavaye, A.R.G. Smith, P.L. Burn, I.R. Gentle, P. Meredith, P.E. Shaw, Diffusion of nitroaromatic vapours into fluorescent dendrimer films for explosives detection, Sensors and Actuators B-Chemical 210 (2015) 550-557.

[5] S. Richardson, H.S. Barcena, G.A. Turnbull, P.L. Burn, I.D.W. Samuel, Chemosensing of 1,4dinitrobenzene using bisfluorene dendrimer distributed feedback lasers, Applied Physics Letters 95(6) (2009). 
[6] S. Shoaee, S.S.Y. Chen, H. Cavaye, A.R.G. Smith, P.L. Burn, I.R. Gentle, P. Meredith, P.E. Shaw, Assessing the sensing limits of fluorescent dendrimer thin films for the detection of explosive vapors, Sensors and Actuators B-Chemical 239 (2017) 727-733.

[7] J. Estella, D. Wencel, J.P. Moore, M. Sourdaine, C. McDonagh, Fabrication and performance evaluation of highly sensitive hybrid sol-gel-derived oxygen sensor films based on a fluorinated precursor, Analytica Chimica Acta 666(1-2) (2010) 83-90.

[8] P.C.A. Jeronimo, A.N. Araujo, M.C.B.S.M. Montenegro, Optical sensors and biosensors based on solgel films, Talanta 72(1) (2007) 13-27.

[9] J.S. Yang, T.M. Swager, Porous shape persistent fluorescent polymer films: An approach to TNT sensory materials, Journal of the American Chemical Society 120(21) (1998) 5321-5322.

[10] H. Sohn, M.J. Sailor, D. Magde, W.C. Trogler, Detection of nitroaromatic explosives based on photoluminescent polymers containing metalloles, Journal of the American Chemical Society 125(13) (2003) 3821-3830.

[11] S.J. Toal, W.C. Trogler, Polymer sensors for nitroaromatic explosives detection, Journal of Materials Chemistry 16(28) (2006) 2871-2883.

[12] A. Alvarez, A. Salinas-Castillo, J.M. Costa-Fernandez, R. Pereiro, A. Sanz-Medel, Fluorescent conjugated polymers for chemical and biochemical sensing, Trac-Trends in Analytical Chemistry 30(9) (2011) 1513-1525.

[13] Y. Salinas, R. Martinez-Manez, M.D. Marcos, F. Sancenon, A.M. Costero, M. Parra, S. Gil, Optical chemosensors and reagents to detect explosives, Chemical Society Reviews 41(3) (2012) 1261-1296.

[14] N. Bolse, R. Eckstein, M. Schend, A. Habermehl, C. Eschenbaum, G. Hernandez-Sosa, U. Lemmer, A digitally printed optoelectronic nose for the selective trace detection of nitroaromatic explosive vapours using fluorescence quenching, Flexible and Printed Electronics 2(2) (2017) 024001.

[15] N. Cennamo, G. D'Agostino, R. Galatus, L. Bibbo, M. Pesavento, L. Zeni, Sensors based on surface plasmon resonance in a plastic optical fiber for the detection of trinitrotoluene, Sensors and Actuators BChemical 188 (2013) 221-226.

[16] A.K.M. Jamil, E.L. Izake, A. Sivanesan, P.M. Fredericks, Rapid detection of TNT in aqueous media by selective label free surface enhanced Raman spectroscopy, Talanta 134 (2015) 732-738.

[17] H. Nie, Y. Lv, L. Yao, Y. Pan, Y. Zhao, P. Li, G. Sun, Y. Ma, M. Zhang, Fluorescence detection of trace TNT by novel cross-linking electropolymerized films both in vapor and aqueous medium, Journal of Hazardous Materials 264 (2014) 474-480.

[18] J. Riedel, M. Berthold, U. Guth, Electrochemical determination of dissolved nitrogen-containing explosives, Electrochimica Acta 128 (2014) 85-90.

[19] N.C. Greenham, I.D.W. Samuel, G.R. Hayes, R.T. Phillips, Y. Kessener, S.C. Moratti, A.B. Holmes, R.H. Friend, MEASUREMENT OF ABSOLUTE PHOTOLUMINESCENCE QUANTUM EFFICIENCIES IN CONJUGATED POLYMERS, Chemical Physics Letters 241(1-2) (1995) 89-96.

[20] Y. Yang, G.A. Turnbull, I.D.W. Samuel, Sensitive Explosive Vapor Detection with Polyfluorene Lasers, Advanced Functional Materials 20(13) (2010) 2093-2097.

[21] R.G. Ewing, M.J. Waltman, D.A. Atkinson, J.W. Grate, P.J. Hotchkiss, The vapor pressures of explosives, Trac-Trends in Analytical Chemistry 42 (2013) 35-48.

[22] X. Sun, Y. Liu, G. Shaw, A. Carrier, S. Dey, J. Zhao, Y. Lei, Fundamental Study of Electrospun PyrenePolyethersulfone Nanofibers Using Mixed Solvents for Sensitive and Selective Explosives Detection in Aqueous Solution, Acs Applied Materials \& Interfaces 7(24) (2015) 13189-13197.

[23] S. Clavaguera, P. Montmeat, F. Parret, E. Pasquinet, J.P. Lere-Porte, L. Hairault, Comparison of fluorescence and QCM technologies: Example of explosives detection with a pi-conjugated thin film, Talanta 82(4) (2010) 1397-1402. 
[24] F.H. Chu, G. Tsiminis, N.A. Spooner, T.M. Monro, Explosives detection by fluorescence quenching of conjugated polymers in suspended core optical fibers, Sensors and Actuators B-Chemical 199 (2014) 2226.

[25] <https://www.cdc.gov/niosh/docs/85-109/>).

[26] J.R. Lakowicz, Principles of Fluorescence Spectroscopy, 3rd ed., Springer US2006.

[27] P. Beyazkilic, A. Yildirim, M. Bayindir, Formation of Pyrene Excimers in Mesoporous Ormosil Thin Films for Visual Detection of Nitro-explosives, Acs Applied Materials \& Interfaces 6(7) (2014) 4997-5004. 